\title{
BLOCKCHAIN READY MANUFACTURING SUPPLY CHAIN USING DISTRIBUTED LEDGER
}

\author{
Saveen A. Abeyratne ${ }^{1}$, Radmehr P. Monfared ${ }^{2}$ \\ ${ }^{1}$ Wolfson School of Mechanical, Electrical and Manufacturing Engineering, Loughborough University, UK \\ ${ }^{2}$ Wolfson School of Mechanical, Electrical and Manufacturing Engineering, Loughborough University, UK
}

\begin{abstract}
The blockchain technology as a foundation for distributed ledgers offers an innovative platform for a new decentralized and transparent transaction mechanism in industries and businesses. The inherited characteristics of this technology enhance trust through transparency and traceability within any transaction of data, goods, and financial resources. Despite initial doubts about this technology, recently governments and large corporations have investigated to adopt and improve this technology in various domains of applications, from finance, social and legal industries to design, manufacturing and supply chain networks. In this article, the authors review the current status of this technology and some of its applications. The potential benefit of such technology in manufacturing supply chain is then discussed in this article and a vision for the future blockchain ready manufacturing supply chain is proposed. Manufacturing of cardboard boxes are used as an example to demonstrate how such technology can be used in a global supply chain network. Finally, the requirements and challenges to adopt this technology in the future manufacturing systems are discussed.
\end{abstract}

Keywords: Blockchain, Distributed Ledger, Manufacturing Supply Chain, Traceability, Digital Currency and Economy

\section{INTRODUCTION}

Marking the dawn of a new era, Blockchain technology is a ground-breaking innovation in decentralized information technology. First invented as part of Bitcoin's underlying infrastructure in 2008 [1], its potential application reaches far beyond digital currencies and financial assets. The technology is still in its early stages and is yet to reach mainstream and enterprise adoption. As the technology gained wider recognition in recent years, there has been a flurry of advancements, new use cases, and applications [2]. The range of potential applications of Blockchain technology is endless, from digital currencies to Blockchain enabled legal contracts [3] with the most promising of applications yet to be developed.

\section{NEED FOR TRANSPARENCY \\ TRACEABILITY IN SUPPLY CHAINS}

AND

There are billions of products being manufactured everyday globally, through complex supply chains that extend to all parts of the world. However, there is very little knowledge of how, when and where these products were originated, manufactured, and used through their life cycle. Even before reaching the end consumer, goods travel through an often vast network of retailers, distributors, transporters, storage facilities, and suppliers that participate in design, production, delivery, and sales, yet in almost every case these journeys remain an unseen dimension of our possessions [4].
Supply chains are getting increasingly more complex, more extended, and more global. An event on one side of the world can stop production or delivery of a service on the other side. The event may be a natural or man-made cause, the event may be large or small, but if the supply of a critical component or service is disrupted, the consequences can be severely harmful to companies further along the supply chain, both financially and in terms of reputation [5].

Additionally, there are many potential negative consequences of the manufacturing industry such as environmental damage, end of life waste, unethical labour and counterfeit products. A sad example of this is the Nike child labour scandal in 1996, where underage children were found employed in factories based in Asia [6]. Another more recent example is the Foxconn suicide scandal in 2010, due to harsh work conditions. [7].

The consumers and end users are often unaware of the various crisis involved with the production of goods. The events such as those mentioned earlier, have enhanced the demand for transparency in manufacturing supply chains as a matter of risk prevention and consumer/labour protection. This is currently developing into a general demand for improved access to information in order to regain consumer trust in products [8].

Sustainability standards and certifications such as Fairtrade, or Organic have become important tools that support conscientious consumption by providing consumers with a better understanding of the product life cycle $[9,10]$. However, the end result is merely a logo of the certification 
printed on products, and consumers are encouraged to accept this information without being able to verify nor completely understand the meaning behind it. Verifying the integrity of the claims made by these certifications is a costly process that requires strenuous auditing. Furthermore, the extension of certification schemes to regions with levels of high corruptions further endangers the credibility of such standards [4]. One recent example of abuse of trust in reputable companies is the VW emissions scandal in 2015 [11] and NISSAN in 2016.

Supply chain visibility is a key business challenge, with most companies having little or no information on their own second and third tier suppliers. End to end supply chain transparency and visibility can help model the flow of products from raw materials to manufacturing, testing, and finished goods, enabling new kinds of analytics for operations, risk and sustainability.

Companies such as Sourcemap, Hiperos and Aravo are taking initiatives to visualize end-to-end supply chains and provide organizations with valuable knowledge on their third party networks [12-14]. However, this revolution of greater transparency in manufacturing supply chains is incremental and mostly voluntary at the moment $[15,16]$.

Transparency enables one to understand the effects and consequences of a decision on a product and furthers understanding of environmental circumstances. However, managing information and control of transparent interactions about every product's supply chain can be a very difficult task. It requires accurate data collection and secure data storage to enable a flow of trusted information between parties. Currently, this responsibility is borne by non-profit, governmental entities or other third parties, through centralized information depositories.

Relying on one single organization to broker such sensitive and valuable information requires a great deal of trust to be invested by every actor in a supply chain. Such organization (as an entity of the manufacturing system) will also gain significant power through the possession of this valuable data, which could be misused to extort or damage organizations if biased. Even if this entity can be trusted to be a good actor, it must possess the technical capabilities to store and handle this information effectively.

A major issue of having this type of centralized system, is that it becomes a single point of failure which leaves the whole system vulnerable to failure (e.g. hacking, or corruption). Various incidents in the past decades have shown that even a tight and costly security mechanism cannot guarantee the complete data security, leaving organizations in a network at potential risk.

Until recently, a centralized system was the most practical approach to achieve data security and controlled transparency in supply chains and services, until the discovery of the Blockchain.

\section{WHAT IS A BLOCKCHAIN?}

Basically, a blockchain could be seen as a distributed ledger: a chronological chain of 'blocks' where each block contains a record of valid network activity since the last block was added to the chain [2].

Each block could be defined as an encrypted piece of information. Theoretically, anyone can add data to the chain of blocks by transacting in the network, anyone can review this data at any time, but no one can change it without adequate authorization.

As a result, a blockchain is a complete and immutable history of network activities, which are shared among all nodes of a distributed network. Blockchain technology for the first time, facilitates two or more entities that may or may not know or trust each other to securely exchange value over the internet without including a third party. Instead, the requirement for validation of transactions is achieved through a process known as 'mining' that ensures the security and validity of the information added to the chain. Blockchain technology can be explained as the technology that powers the Internet of Transactions [17].

A significant property of blockchains is that it operates on a decentralized network meaning there is no single entity that controls or governs the system. Eliminating the need for third party intermediation or control facilitates towards removing friction in all types of value exchange that can arise in the form of costs, risk, information and control [2].

Another powerful characteristic of a blockchain is its ability to get a distributed network to reach consensus regarding the state of data and agreeing on the rules of the network without a central governing entity. Consequently, improvement to a system can be proposed by any user, but are implemented only if accepted by all parties involved in the network, hence, enhanced transparency and trust. In a typical blockchain interaction, trust among a distributed network is possible due to the validation or mining process where each new transaction is verified by the whole network before being added to a blockchain.

Mining is the process of adding new blocks of data onto the ongoing chain through validation by each node on the network known as 'miners'. A miner adds each new block on the chain after solving a cryptographic algorithm, which must be accepted by majority or all (based on the definition of the block) of the nodes in the network as a valid data. The network rewards the miner for adding a valid block to the chain by some form of digital credit. This credit is the primary incentive for miners to constantly validate and maintain the consistency of data throughout the network. The reward will be in the form of financial gains, or an approval for completion of an event once the block is accepted by the whole network. Miners are independent entities in a network and no single miner is capable of changing, or adding invalid data without being detected by the rest of the network as a 'bad actor'. When the network rejects a block, the miner does not receive the network 
reward, however the rejected block is logged in the system, allowing the network to recognize the miner as a possible threat. This method significantly improves the traceability in a system.

\subsection{Technological Advantages of Blockchain}

\section{Technology}

Blockchain inherently provides several key technological advantages to users that are implications of its structural architecture. Some of which including durability, transparency, immutability and process integrity are described below.

Durability - Decentralized networks eliminate single points of failure as opposed to centralized systems. This distribution of risk among its nodes makes blockchains much more durable than centralized systems and are better suited to deter malicious accesses.

Transparency - An identical copy of a blockchain is maintained by each node on the network, allowing auditing and inspecting of the data sets in real time. This level of transparency makes network activities and operations highly visible, thereby reducing the need for trust.

Immutability - Data that is stored on a distributed public blockchain is practically immutable due to the need for validation by other nodes and traceability of changes. This allows users to operate with the highest degree of confidence that the chain of data is unaltered and accurate [2].

Process Integrity - Distributed open source protocols are by nature executed exactly as written in the code. Users can be certain that actions described on the protocol are executed correctly and timely without the need for human intervention.

\section{RAPID GROWTH IN NEW APPLICATION DOMAINS}

Over seven years since the discovery of blockchain technology, this field has seen massive growth through various new innovative and technological concepts put forward. Initially this technology gained a negative reputation due to its association with untraceable purchases on the 'dark net' where users would use digital currencies such as Bitcoin to make anonymous purchases. However, the recent years have seen many large companies such as IBM, JPMorgan and Barclays investing in the research and development of the Blockchain technology [18-20].

Due to such interest from major organizations, and the large amount of money being circulated in cryptocurrencies, innovative and creative organizations and entrepreneurs have been attracted to this new field of information technology. This rapid growth in the field has changed the perspective of many governments to see the potential of this technology over its initial affiliation with illegal activities
[21]. Some existing applications and use cases of blockchain are summarized below.

\subsection{Financial Applications}

Currencies - Digital currencies such as Bitcoin were the first use case of blockchain technology, offering a fully decentralized issuance of currency, and traceable payments. Following Bitcoin's growing success, a large number of digital currencies have been created, which are variations of the Bitcoin system architecture. Currently there are over 600 different digital currencies that use blockchain technology as their underlying technology layer [22]. Digital currencies remain the most popular use case of blockchain technology, however advancements and innovations in this field, have led to numerous other use cases.

Exchanges - Blockchain can be used to create decentralized systems, which facilitate the exchange or digital currencies such as Bitcoin, or the exchange of any other form of asset that can be registered with its own digital identity on a network. Companies such as Coinbase, ItBit or Kraken are examples of digital currency exchanges that currently exist [23-25].

Stock Market - Decentralized stock markets [26] can be powered though blockchain technology, where the stocks can be traded on a platform that is not controlled by any single governing body as opposed to current systems. Users can be certain that the exchanges are carried out correctly since the system will only function as described by the system protocol. However, this application has not been adopted yet.

\subsection{Social Applications}

Digital Identity - Blockchain technology could provide the infrastructure to scale digital identity [27] at extremely low costs with significant improvements in security. Instead of various governments issuing identities or passports to citizens, a decentralized identity service on using blockchain technology can provide users from all over the world to obtain their own digital identity through a decentralized system. This application had attracted attention of many governmental organizations.

Voting - The blockchain technology through the use of "private keys" for each voter can be used to authenticate the voting process. In this application, the system protocol can be designed such that the identities of the users can be validated but kept anonymous while calculating the final result of the election in real-time. Since the protocol is transparent, voters can be certain that the results are accurate and not vulnerable to manipulation and fraud.

\subsection{Legal Applications}

Smart Contracts - Blockchain based smart contracts are an emerging use case of blockchain technology [28]. The idea of smart contracts is relatively straightforward: A software protocol performs an action (releases funds, sends information, makes purchase, etc.) when certain conditions 
are met (a payment is received, the outcome of an event is determined, etc.). The advantage of blockchain-based contracts is that they reduce the amount of human involvement required to create, execute and enforce a contract, thereby lowering its cost while raising the assurance of execution and enforcement processes.

Smart Property - The general concept of smart contracts is the notion of transacting all property in blockchain-based models [29]. Digital identities can be created for any physical world hard asset that is represented in the blockchain system. Using these identities, ownership can be controlled through smart contracts, for example the room door in a hotel room may be unlocked automatically when the user's payment is accepted, or a car that doesn't allow the user to drive, if their insurance is expired.

\subsection{Other Potential Applications}

Asset Tracking - Blockchains can be used to trace physical assets which allowing a record of ownership to be maintained for each asset. For example, Everledger is a company that tracks diamonds through creating a digital identity for each diamond on a blockchain network [30]. This aids the authentication of the transaction, for example by preventing what is known as "blood diamonds" entering the jewelry market. Another example of asset tracking is provided by Provenance Company, which focuses on creating a chain of custody or a record of ownership for various physical products [31].

Engineering Applications - Complex engineering systems are deemed to be the next potential application domain for the Blockchain technology. The authors envisage a number of engineering related transactions that can immediately benefit from the blockchain approach. These include: controlling financial transactions between and within companies, asset and product tracking during and after manufacturing, controlling the quality and the tests, distributed modular designs for machines and systems, and the manufacturing supply chain, which is the main focus of this article.

\section{MANAGING MANUFACTURING SUPPLY CHAIN USING BLOCKCHAIN TECHNOLOGY}

Supply chain in manufacturing systems comprises a series of system entities including people, physical resources, knowledge, processes, and financial contracts and transactions that facilitate moving a product from supplier to customer. In a large supply chain system, it is very difficult to have an overall picture of all transactions within the chains [32]. This information is typically stored in multiple locations and are accessible to certain system entities. In such systems. the customers (being the final consumer or the larger company within the chain) usually have partial access to the overall information [33]. In many cases, part of the information is treated as a commodity for a supplier. Therefore, due to the low level of transparency, the tractability of transactions is based on the trust between the system actors.
The blockchain technology can potentially improve the transparency and traceability issues within the manufacturing supply chain through the use of immutable record of data, distributed storage, and controlled user accesses.

A decentralized distributed system that uses Blockchain technologies to collect, store and manage key product information of each individual product throughout its life cycle is proposed in this article. Such distributed block of information potentially creates a secure, shared record of transactions for each individual product along with specific product information.

The proposed system is explained in the following sections, including the types of actors in a network of suppliers, how they access and register themselves in the network, and how data entry is authorized, validated, and stored.

\subsection{Blockchain Ready Manufacturing Supply Chain}

An overview of proposed application of blockchain in supply chain for manufacturing systems is illustrated by Fig1. The proposes approach comprises of a decentralized distributed system that uses blockchain(s) to collect, store and manage key product information of each product throughout its life cycle. This creates a secure, shared record of exchange for each product along with specific product information.

As a product moves through its life cycle, it is possessed by a variety of actors, for example, producers, suppliers, manufacturers, distributors, retailers and finally the end consumer. Each of these actors play an important part in this system, logging in key information about the product and its current status on to the blockchain network. Each product would have a unique digital profile containing all related information, populated during various life cycle stages.

Each product would be attached with an information tag, which could be in the form of a barcode, RFID or QR code. This tag represents a unique digital cryptographic identifier that links a physical product to its virtual identity on the network. This virtual identity is presented on the system software as a part of the product digital profile.

Actors will also have their own digital profile on the network, which is created upon registration. This profile displays information such as their description, location, certifications and association with products. A product that has been signed by an actor would have a link from the product profile to the actor's profile. The system allows actors to change the privacy of their profile to different types of actors. Actors can choose to remain completely anonymous, however must be certified by a registered auditor or certifier to maintain the trust in the system. 


\section{Blockchain enabled Manufacturing Supply Chains}

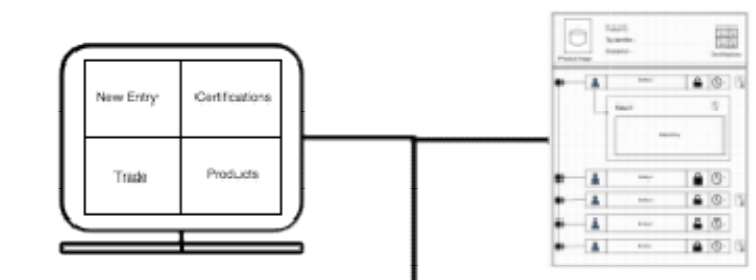

Data Entry - for Organisations

Data Access - Product Proflile

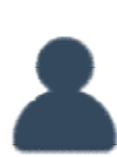

USER

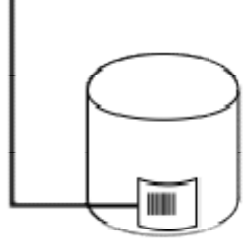

Product with Tag

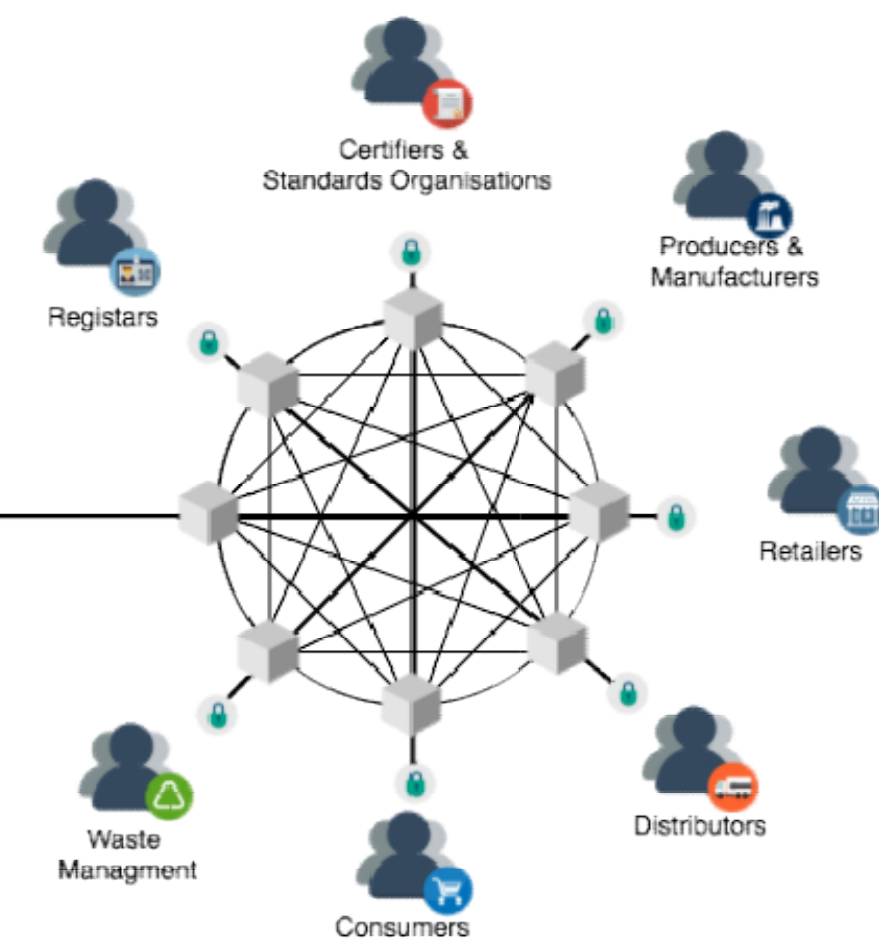

Blockchain

Authenticated Access

Fig-1: Overview of the proposed concept

The system actors register themselves on the network through a registrar, which is an accreditation service that provides credentials and a unique identity to the actors. Upon registration, a public and private cryptographic key pair are generated for each actor. The public key identifies the actor within the network and the private key authenticates the actor when interacting with the system. Actors can only interact with the network by cryptographically authenticating themselves using their private key. This allows each product to be digitally signed by the actors when being exchanged or added to further down in the supply chain.

This system consists of several types of actors:

Registrars - provides unique identities to actors on the network.

Standards Organizations - defines standards schemes (such as Fairtrade)

Certifiers - provides certifications to actors, which allow them to participate in the network.
Producers, manufactures, distributors, retailers and waste management organizations - enter key product specific data to the blockchain

Consumers - purchases products, and in some cases are allowed to enter product data to the blockchain.

Each actor using this system accesses the specific network of blockchain through a user interface. The software application used by the actors is configured for specific digital profile of a product. The system software is developed by a group of trusted parties, and is accessible for registered organizations and institutions to download and run on their systems. Consumers would have customized version of the user interface to allows access data about a product they are associated with. The system software facilitates the entry of new data and the access to the existing data. This software is run on a blockchain that allows programmable code to be executed, such as the Ethereum Blockchain [3].

All data in the network is stored on a blockchain and is available to anyone running the system software with a 
correct authentication. The data access given to actor changes depending on their type and position in the supply chain. The set of rules that govern the system is written in code and stored in the blockchain. These rules define how the actors in the network are to interact with the system, and how the data will be shared among the network.

The rules cannot be changed by the actors and therefore guarantee integrity and the conditions for the validity of data. Once the rules are stored on the blockchain, they function exactly as defined and cannot be altered without broadcasting to all nodes and verification by key actors.

Certifications and standards programs for example, Fairtrade or FSC, can be implemented in this system [9, 34]. Certifiers and auditors in the network will visit the factories and facilities to inspect if the rules for standard programs are being met. Once verified by the certifiers, the actor's profile and its products can be digitally signed by the certifiers and standards organization, to prove their certification. All actors are inspected by the certifiers to verify their identity. The certifiers must disclose all actors' identity to the network through a registrar. This is to maximize the transparency of system elements, while maintaining the integrity and security of data.

\subsection{Data Entry}

Each physical product in proposed system has to be presented digitally on a blockchain network, so that all stakeholders of that product will have direct access to that product profile. This is necessary to enable trades and updating product information.

Using the digital identities of the actors and products, it is possible for a "smart contract" to be created for each product in form of rules, so that only the parties with the correct digital keys have access to that product. At a given time, a product is 'owned' by a particular actor. Only this actor has the permissions to enter new information into that product's profile or initiate a trade with another party. Therefore, when the product is transferred (or sold) to another actor, both parties must sign a digital contract to authenticate the exchange. Once all parties have signed the contract, the details of the transaction will be added to the blockchain. The network will process this data and update the status of that product profile, showing its new stakeholders. This allows the network to maintain an indisputable record of ownership for each product. When the exchange is completed, the system updates the permissions such that only the new owner can create a new entry and update the product's details. Since both parties have to prove their identity by signing with their private key, the details entered onto the profile is guaranteed to have been entered by the respective party.

The data could be added either manually or automatically by the actors, who must authenticate themselves using their private key to connect to the network. A software application provides the actors with an interface to create new entries for products. There is a range of data that could be collected in this system. They could vary depending on the status of the product, the type of product, and the standards that are to be implemented for this product. The records of data transactions would be automatically updated by the system when an exchange is initiated. Additional product specific information is added by the owner of the product when they are given access to create a new entry.

Below are several types of data that can be collected with regard to a certain product.

Ownership data - chronological list of all previous owners of the product or entity in the blockchain network including the current owner. Each time the product is exchanged between two parties, a new entry is created by the system recording the details of the transacting parties and is added to the product's profile. The transacting parties are referred to by their digital identity. Thereby enabling the system to assign data entry permissions to the correct party. Furthermore, this enables implementing a controlled level of transparency amongst related parties.

Time stamping - When a new entry is created on a product's profile, the system automatically records the time of that entry. This allows the network to create a chronological order of entries related to that specific product.

Location data - Where the product has been and where is it now currently are recorded using the location data. Since the system has location details of all the registered actors, it can record the location of a product every time an actor makes a new entry. The location information may be simply a unique location ID, or dynamic GPS data which could be implemented for certain supply chains.

Product specific data - This is the key information that is specific to a product. This information can be used to prove certain attributes of the product or provide performance data as feedback to producers, manufacturers, and quality controller.

Environmental impact data- Additional information regarding the environmental impact of the product through its life cycle (e.g. energy consumption, $\mathrm{CO} 2$ emission).

Certain products could be programmed to transmit their performance data to the blockchain during their working life span. For example, a mobile phone could update information about its battery performance to the blockchain during its use. This information could then be accessed by the respective battery manufacturers and designers, to improve their product based on such feedback. Another example of this would be a vehicle that is connected to the Internet, which autonomously uploads performance and environmental impact data to the blockchain. This information can be very helpful to the future buyer of this vehicle, as they would have access to the vehicle historic performance data, so they can make more informed decisions. At the same time, the engine designers and the vehicle manufacturers can also benefit from this information. 


\subsection{Data Access}

The products' digital profile acts as the primary source of accessing product information for all actors in this system. Fig 2 shows an example template of a product profile.

Each entry is time stamped and digitally signed by the actors. The certifications affiliated with the product are also recorded in the digital profile of a product (as shown in the figure). To maintain control over the user accesses to the information within a profile, including the specific product details and identities of actors, certain rules would be embedded into the product's profile. All actors are required to authenticate themselves using their private keys when accessing the profile of a product. This allows the system to display the profile, according to the permissions set to that actor. Fig 2 indicates the access as a locked or unlocked sign for each entry, which represents the level of permissions granted for each different user accessing this profile.

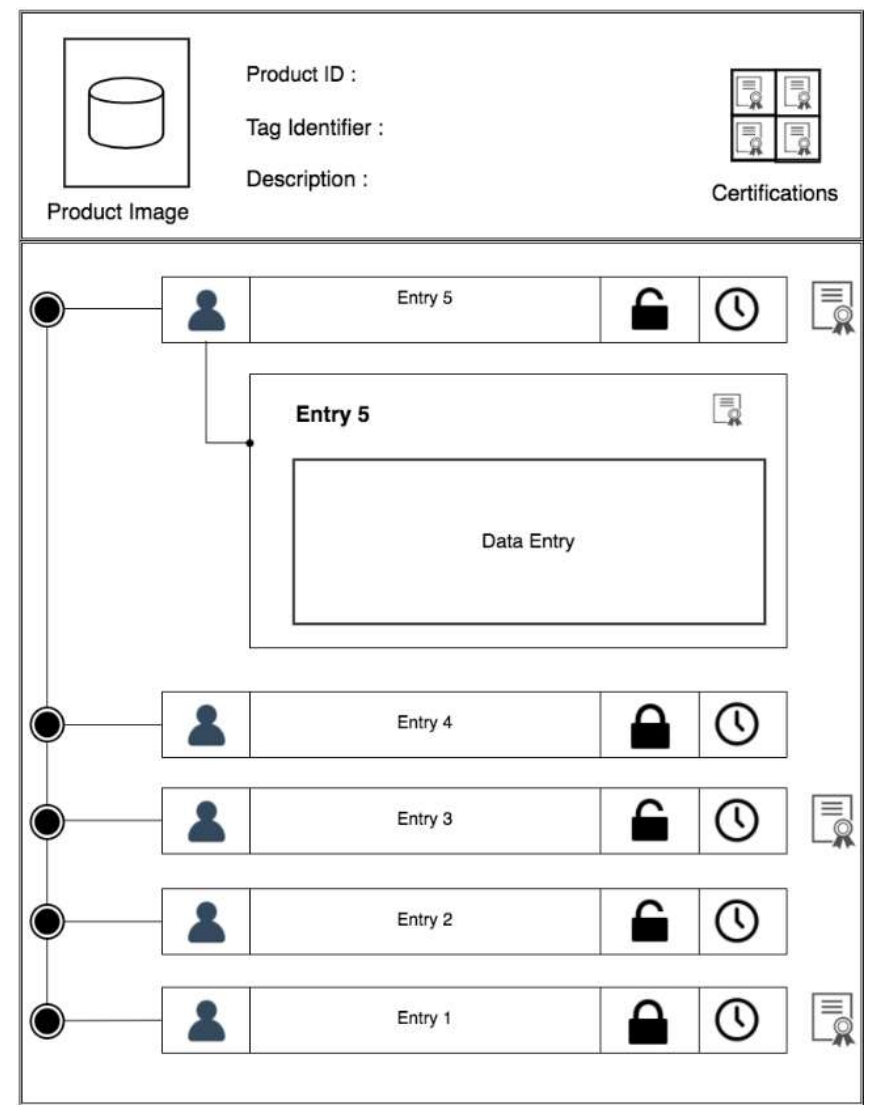

Fig-2: Example template for a product profile

For example, a food product such as banana would have a profile that contains details such as when the fruit was harvested, when it was distributed and where it was farmed. A consumer purchasing that item would have access to these details but not the specific names of the farm that the fruit was produced in. Whereas, a distributer or quality controller would have access to more information such as the identity of farm, details of the weather conditions during that harvest, or the storage temperatures.

\section{APPLICATION SCENARIO}

In this section an example application scenario is explained to better clarify the potential for the proposed concept. The application of the blockchain ready supply chain is considered for the raw material, manufacturing, distributing and recycling of a cardboard box.

There are a large number of actors involved in the manufacturing and the supply chain of a cardboard box. The application of blockchain in this example could extend not only to the manufacturing of the box, but the product packed in the box.

In this scenario we focus only on a single branch of the supply chain, which includes the raw material extraction for paper, its transformation to a cardboard box, its use as packaging and finally the recycling stage as depicted by Fig 3.

All actors mentioned in this scenario, have registered themselves in the system through a registrar service, and has their own unique identity on the network, including an actor profile. The actors interact with the system using the interface created by the system software for organizations. In a blockchain ready cardboard box manufacturing scenario, the business processes would be involved.

1. Forestry - The trees cut down at the forestry are labelled with tags and are entered in to the blockchain as new products. Key information specific to the tree such as its type and age could be entered to the system. A new trade is initiated between the forester and the paper manufacturer, where the goods are exchanged after signing a digital contract that stored on the blockchain (the data entry would be through handheld tag readers).

The forester is certified by a standard organization who appoints certifiers to check if the regulations have been met (e.g. if the trees are cut without destroying natural forests). This certificate is shown on the actor's profile with digital signatures of both the certifiers and the standard organizations.

2. Paper Manufacturer - The trees are accepted by the paper manufacturer by scanning them through their network connected scanners. This closes the exchange and gives the paper manufacture access to read and enter new data onto the product's profile. Here, other raw materials are combined with the wood to create paper, which on completion will have a new tag (or modify the existing one - if rewritable tag used). During the operation, the tags of the raw materials are destroyed, but their product's profiles are updated and show their presence in the resulting papers' profile. Further physical tag can be produced and attached to the product after completion of the process. 
1

2

3

4

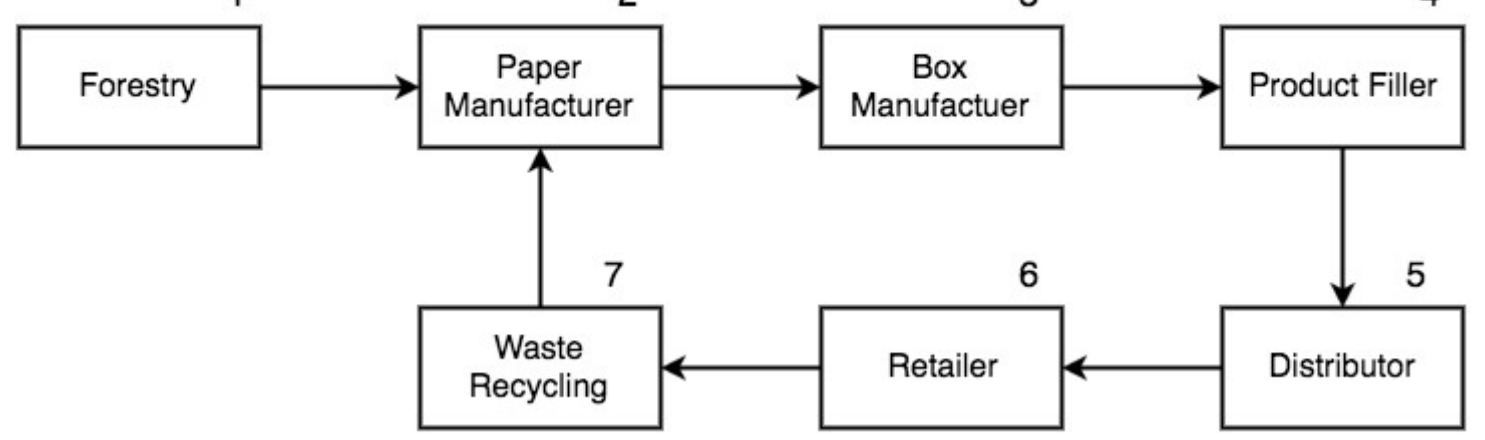

Fig-3: Part of the manufacturing supply chain for a cardboard box

3. Box Manufacturer - The paper rolls are sold to the box manufacturer who accepts the goods by digitally signing a contract, thereby gaining access to the product's profile. The box manufacturer enters key new information such as the quality of the box, strength test results and paper combination. This product is sold to the next actor in the supply chain after the product data has been entered.

4. Product Filler - The product filler uses the boxes to package their own product. The product profile of this product is updated along with that of the box, to show that they are both one item, with two different tags. This allows the product inside a box to be recognized by scanning the box's tag. This is crucial for the distribution system.

5. Distributor - The next actor in this supply chain is the distributor, who transports the product through various locations in various containers. At each of these locations, the profiles of the products are updated by scanning the tag of the larger container or pallet that contains multiple packed boxes. This makes it easier for products to be updated quickly and efficiently. The blockchain related to the larger container will automatically updates the subsequent chains to enable the traceability of an item in a larger batch.

6. Retailer - The retailer accepts the goods as filled boxes of product, and removes the products from the boxes and updates the profiles of the boxes with its current state. These boxes have now served its purpose and are now sent for recycling by the retailer.

A trade is initiated with the waste recycler who accepts the waste boxes by digitally signing a contract on the blockchain. The product digital profile would now be separated from the batch and the box in the blockchain, however the record of all previous stages will remain on the blockchain.

7. Waste Recycler - The recycler processes the cardboard boxes into batches of used paper packaging and creates a new identity on the network. The recycler then enters key information about the recycled product and initiates a trade with a paper manufacturer. The paper manufacturer uses this recycled paper as part of its paper production, thus closing the loop in the life cycle of a cardboard box. However, the raw material can still be traced to the next box produced, hence a comprehensive record of data can be maintained.

\section{CONCLUSION}

Blockchain technology is a revolutionary innovation with capability to transform many existing traditional systems into more secure, distributed, transparent, collaborative systems while empowering its users.

In this article, the authors reviewed some of the main characteristics of the Blockchain technology and discussed potential application domains. Then the focus of the article was placed on the application within the supply chain in manufacturing businesses. A hypothetical vision for a blockchain ready manufacturing supply chain was proposed and an example scenario for part of a supply chain for production of cardboard boxes was explained from manufacturing to recycling.

In this article an idea has been put forward to demonstrate potential benefits and stimulate further research. The authors fully understand the cultural and technical challenges ahead. However, it is believed that the potential benefits to the consumers, suppliers, and the impact on the environment provide sufficient motivation to progress.

The proposed system facilitates a vast amount of data to be collected about the products and users in manufacturing industry, which can prove to be beneficial to a range of different people, organizations, governments and researchers. For instance, this allows consumers to readily access accurate data specific to any product that has been manufactured through a blockchain enabled supply chain, thus allowing them to make better buying decisions. Organizations involved in the design, manufacturing and production can gain an improved understanding of how their products are used further along the supply chain. This level of feedback can be used to improve their technology and 
marketing, as well as their production accounting and sales accounting. The integration of smart contracts into this system can improve the security of transactions as each item can only be received by the buyer who has signed the relevant contract with the seller; allowing the system to identify fraudulent transactions or misplaced items.

However, this technology requires certain IT infrastructure in place for all actors, such as access to the Internet, which may be impractical at the moment for some remote providers of raw material. The digital profiles would need to be kept updated constantly through manual or automated systems such as simple or RFID tags. Smart contracts should be implemented and be embedded into the system to provide incentives to enable blockchain to govern progress of a business process. In addition, performance capability of such system may be proved to be a bottleneck for the implementation of proposed solution.

It would be necessary that the large manufacturing organizations be attracted to the potential benefits of the proposed supply chain systems. They may be reluctant initially to characteristics such as lifecycle transparency and automated payments, as occasionally these are used as a business leverage. However, the experience from digital currencies and large financial institutes has proved that large industrial and governmental organizations will see the potential and try to remain open-minded towards this technology.

Much research is required to develop this idea for application at an industrial scale with close collaboration between research, industrial, and governmental organizations.

\section{REFERENCES}

[1] S. Nakamoto, "Bitcoin: A Peer-to-Peer Electronic Cash System," Consulted, pp. 1-9, 2008.

[2] S. Bogart and K. Rice, "The Blockchain Report: Welcome to the Internet of Value," 2015.

[3] V. Buterin, "A next-generation smart contract and decentralized application platform," Etherum, no. January, pp. 1-36, 2014.

[4] B. Jessi, S. Jutta, and G. Wood, "Provenance White Paper," 2016. https://www.provenance.org/whitepaper.

[5] A. Dr. Punter, "Supply Chain Failures," 2013. [Online]. http://www.airmic.com/sites/default/files/supply_chain failures_2013_FINAL_web.pdf

[6] $\bar{C}$. Tim, Still Waiting For Nike To Do It. San Francisco: Gobal Exchange, 2001.

[7] M. Moore, "Mass suicide' protest at Apple manufacturer Foxconn factory," The Telegraph, UK, 2012.

[Online].

http://www.telegraph.co.uk/news/worldnews/asia/china /9006988/Mass-suicide-protest-at-Apple-manufacturerFoxconn-factory.html

[8] F. Mechthild, T. Ludwig, "Transparency in Supply Chains: Is Trust a Limiting Factor?" 2006. http://ageconsearch.umn.edu/bitstream/7733/1/sp06fr01 .pdf
[9] S. D. Elder, H. Zerriffi, and P. Le Billon, "Is Fairtrade certification greening agricultural practices? An analysis of Fairtrade environmental standards in Rwanda,” J. Rural Stud., vol. 32, pp. 264-274, 2013.

[10] A. Baier, "Organic Certification Process," Review Literature And Arts Of The Americas. p. 8, 2005.

[11] E. Taylor and A. Cremer, "Volkswagen takes \$18 billion hit over emissions scandal | Reuters," 2016. [Online]. http://uk.reuters.com/article/us-volkswagenemissions-germany-probe-idUKKCN0XJ19U

[12] L. Bonanni, "Sourcemap: eco-design, sustainable supply chains, and radical transparency," XRDS Crossroads, ACM Mag. Students, vol. 17, no. 4, pp. 22-26, 2011.

[13] F. David, "Microsoft Procurement Moves to the Cloud with Hiperos | Business Wire," Business Wire, 2010. http://www.businesswire.com/news/home/2010090100 5217/en/Microsoft-Procurement-Moves-Cloud-Hiperos

[14] J. Busch, “Aravo: The Last of the Independent Supplier Information Management Mohicans? - Spend Matters Spend Matters," SpendMatters, 2010. [Online]. http://spendmatters.com/2010/10/12 /aravo-the-last-ofthe-independent-supplier-information-managementmohicans/

[15] D. Linich, "Path to Supply Chain Transparency," Deloitte University Press, 2014. [Online]. http://www.deloitte.co.uk/makeconnections/assets/pdf/ path-to-supply-chain-transparency.pdf

[16] S. New, "Preventing Another Bangladesh Tragedy: Three Ways to Transform Supply Chain Ethics," Havard Business Review, 2013. [Online]. https://hbr.org/2013/05/preventing-another-bangladesh

[17] M. Mainelli and A. Milne, "The Impact and Potential of Blockchain on the Securities Transaction Lifecycle," 2016. Available http://www.zyen.com/now-andzyen/blog/ 1516-the-impact-and-potential-ofblockchain-on-the-securities-transaction-lifecycle.html

[18] G. Chavez-Dreyfuss, "IBM launches new cloud services for blockchain | Reuters," Reuters, 2016. [19]

E. Glazer, "JPMorgan Quietly Tests 'Blockchain' With 2,200 Clients - WSJ," The Wall Street Journal, 2016.

[20] S. Taylor, "Blockchain: understanding the potential." 2015.

[21] UK Government, "Distributed Ledger Technology: beyond block chain," 2016. [Online]. https://www.gov.uk/government/uploads/system/upload s/attachment_data/file/492972/gs-16-1-distributedledger-technology.pdf

[22] L. David, "The Future Of Canadian Currency May Be Digital | David Leonhardt," The Huffington Post Canada, 2016. [Online]. http://www.huffingtonpost.ca/david-leonhardt/digitalcurrency-cryptocurrency_b_9768432.html

[23] P. Vigna, "Coinbase Raises \$75 Million in Funding Round - WSJ," The Wall Street Journal, 2015. [Online]. http://www.wsj.com/articles/coinbase-raises75-million-in-funding-round-1421762403

[24] Oscar Williams-Grut, "New York's itBit is trying to bring the tech behind bitcoin to Wall Street — here's why," Business Insider UK, 2016. [Online]. 
http://uk.businessinsider.com/interview-itbit-ceo-chadcascarilla-blockchain-2016-2

[25] L. Parker, “Kraken's business booms, as Money Partners Group joins SBI Investment in 'multi-million dollar' investment» Brave New Coin," Brave New Coin, 2016. [Online]. http://bravenewcoin.com/news/ krakens-business-booms-as-money-partners-groupjoins-sbi-investment-in-multi-million-dollarinvestment/

[26] I. Allison, "Overstock's Patrick Byrne: I feel the blockchain revolution is my victory," International Business Times, 2016. [Online]. http://www.ibtimes.co.uk/overstocks-patrick-byrne-ifeel-blockchain-revolution-my-victory- 1554518

[27] J. Maxim, "Onename Launches Blockchain Identity Product Passcard," Bitcoin Magazine, 2015. [Online]. https://bitcoinmagazine.com/articles/onenamelaunches-blockchain-identity-product-passcard1431548450

[28] Bitfury Group, "Smart Contracts on Bitcoin Blockchain," 2015.

[29] M. Swan, Blockchain : Blueprint for a New Economy. O’Reilly Media, 2015.

[30] Rob Price, "This London startup could make diamond theft a thing of the past — and that's just the start," Business Insider UK, 2015. [Online]. http://uk.businessinsider.com/everledger-ledgerdiamonds-blockchain-tech-theft-fraud-2015-8

[31] G. Greenspan and M. Zehavi, "Will Provenance Be the Blockchain's Break Out Use Case in 2016? CoinDesk," CoinDesk, 2016. [Online]. http://www.coindesk.com/provenance-blockchain-techapp/

[32] Haq, I., Monfared, etal, A., "A new vision for the automation systems engineering for automotive powertrain assembly," International Journal of Computer Integrated Manufacturing (IJCIM), vol. 23, pp. 308-324, 2010.

[33] Monfared, R.P., Enterprise Modelling: A ComponentBased Approach to Design and Construction of Change Capable Manufacturing Cell Control Systems, 1st ed. vol. 1. Saarbrücken-Germany: VDM Verlag, 2009. ISBN 3639177568.

[34] Forest Stewardship Council A.C., "FSC International Standard. FSC Principles and Criteria for Forest," FSC Stand., vol. 01, no. 001 (verssion 4-0), pp. 1-13, 1996. 